\title{
Study of the conditions of fracture at explosive compaction of powders
}

\author{
Andrey E. Buzyurkin, Evgeny I. Kraus \\ Khristianovich Institute of Theoretical and Applied Mechanics of Siberian Branch of RAS, 4/1 Instituskaya str., Novosibirsk, \\ 630090, Russia \\ buzjura@itam.nsc.ru, kraus@itam.nsc.ru \\ Yaroslav L. Lukyanov \\ Lavrentyev Institute of Hydrodynamics of Siberian Branch of RAS, 15 Lavrentyev pr., Novosibirsk, 630090, Russia \\ lukyanov@bydro.nsc.ru
}

\begin{abstract}
Joint theoretical and experimental investigations have allowed to realize an approach with use of mathematical and physical modeling of processes of a shock wave loading of powder materials.

In order to gain a better insight into the effect of loading conditions and, in particular, to study the effect of detonation velocity, explosive thickness, and explosion pressure on the properties of the final sample, we numerically solved the problem about powder compaction in the axisymmetric case.

The performed analysis shows that an increase in the decay time of the pressure applied to the sample due to an increase of the explosive thickness or the external loading causes no shrinkage of the destructed region at a fixed propagation velocity of the detonation wave. Simultaneously, a decrease in the propagation velocity of the detonation wave results in an appreciable shrinkage of this region.
\end{abstract}

KEYwORDS. Shock waves; Fracture; Powder.

\section{INTRODUCTION}

$\mathrm{M}$ ethods of explosive loading of powder materials in conservation ampoules are applied in order to obtain new materials including composite ones with the unique physical and mechanical properties. In addition, these methods can be used to study phase transitions occurring in materials at high pressures and temperatures taking place behind shock waves, as well as for the synthesis of metastable phases.

In recent decades, significant development has been achieved in such a scientific and technical branch of materials science as powder metallurgy. This term is currently understood a whole complex of problems connected with the design of materials and products from metal and nonmetal powders. Interest in these problems is quite understandable since the opportunity to create new classes of materials with unique and controllable properties which can not be obtained by ordinary metallurgy methods has arisen.

A special place in the powder metallurgy is occupied by explosive compaction of powder materials. It is easy to explain the strong interest in the explosive compaction. It consists in the fact that virtually all the methods of composite materials' production from powder mixtures lead to a change in initial material properties due to high temperatures and relatively long duration of the process. 
Since the powders being in the form of granules, fibers, needles and ribbons, possessing the necessary properties in the initial state, can not be used directly to produce semi-finished products or components, the methods of compaction of these materials perform two tasks at once. On the one hand the compaction changes the shape and size of the powders, and on the other hand it produces the material itself. From this point of view, the short exposure to high temperatures and pressures during explosive compaction allows, in general, to keep the original structure and properties of the components. At the same time, varying of the intensity and time exposure to high pressure and temperature in shock compression allows to modify, if necessary, the structure and properties of the compacts a controlled manner.

The loading of the powder materials in the conservation ampoules can be carried out by means of both plane and oblique shock waves. Each of the methods has its pros and cons. The explosive loading by oblique shock wave is characterized by high values of shear strain, in comparison with the plane impact, which leads to stronger bonds between the compacted particles. In addition, this scheme allows to obtain the compacts not only in the form of plates, but pipes, rods, cones, etc as well. One can also get the compacts of large sizes. The loading by plane shock waves allow to vary the pressure and temperature behind the shock front in a wider range and to reach much higher values of these parameters. At the same time, the method is more material-consuming and has limitations on the size of the loaded samples.

Investigation into the interaction between oblique shock waves in porous materials and powders is a topical problem in optimization of loading conditions for obtaining, from a given sample, a compacted material with spatially uniform physical and mechanical properties. In compacting a powder in the cylindrical scheme, an irregular interaction between shock waves occurs. The compacted powder displays substantial non-uniformity in particle displacements, resulting in inhomogeneity of powder characteristics and, in some cases, even in material failure.

In compacting porous material and powders, the strong bonding between particles is achieved through the combined pressure-shear loading. During the compacting, a substantial energy is released at the interfaces between powder particles, resulting in surface cleaning and material melting in narrow interfacial regions. As a result, pore collapsing, giving rise to strong bonding between particles, occurs. Below, this phenomenon is termed compaction.

V.F. Nesterenko proposed the following criterion for the formation of a strong compact:

$$
P>2 H_{V}
$$

where, according to [1], $H_{V} \approx 3 Y_{s}$. Following [1], we can write criterion (1), deduced from experimental data, as

$$
P>6 Y_{s}
$$

In turn, R. Prummer [2] uses the following condition for obtaining a uniform, in its physical properties, cylindrical compact with no Mach reflection induced singularities at its center: $P \approx H_{V}$, where $P$ is the detonation pressure. Comparing condition (1) with the condition $P \approx H_{V}$, Nesterenko [1] arrives at a conclusion that it is impossible in principle, without a central rod, to obtain a spatially uniform compact in the cylindrical loading scheme since the shock pressure required for obtaining a dense compact (2) will always lead to Mach reflection at the center of the sample.

Another important problem is preservation of the finish compact after loading. With the arrival of unloading waves, there arises a tensile stress that results in partial or complete destruction of the sample. We assume that the sample undergoes mechanical failure if the maximum tensile stress $\sigma_{\max }$ reaches a certain critical value $\sigma_{*}$. In line with the adopted hypothesis, the following condition for the sample failure should be assumed:

$$
\sigma_{\max }>\sigma_{*}
$$

where $\sigma_{\max }$ is the highest stress among the principal stresses for the strained state under study and $\sigma_{*}$ is the critical stress.

In the present work, the critical stress $\sigma_{*}$ is estimated as

$$
\sigma_{*}=(2 / 3) Y_{s} \ln \left(1 / m_{1}\right)
$$

where $m_{1}$ is the residual porosity. Taking the finish-compact density to equal $99 \%$, we obtain $m_{1}=0.01$ and $\sigma_{*} \approx 3 Y_{s}$. For the principal stresses, we have:

$$
\begin{aligned}
& \sigma_{1}=\frac{\sigma_{x x}+\sigma_{y y}}{2}+\frac{1}{2} \sqrt{\left(\sigma_{x x}-\sigma_{y y}\right)^{2}+4 \sigma_{x y}^{2}} \\
& \sigma_{2}=\frac{\sigma_{x x}+\sigma_{y y}}{2}-\frac{1}{2} \sqrt{\left(\sigma_{x x}-\sigma_{y y}\right)^{2}+4 \sigma_{x y}^{2}}
\end{aligned}
$$




$$
\sigma_{3}=\sigma_{\theta \theta}
$$

\section{EXPERIMENTAL STUDY OF THE COMPACT STRUCTURE}

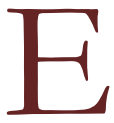
xperiments on the explosive compaction have been conducted by a cylindrical scheme without a central rod. The powder has consisted of particles of nearly spherical shape and with size of $145-310 \mu \mathrm{m}$ (Fig. 1). Bulk density of the powder has been in all experiments for copper equal to $-5.0 \pm 0.05 \mathrm{~g} / \mathrm{cm}^{3}$, for aluminum $-1.4 \mathrm{~g} / \mathrm{cm}^{3}$.

The experimental arrangement is shown in Fig. 2.

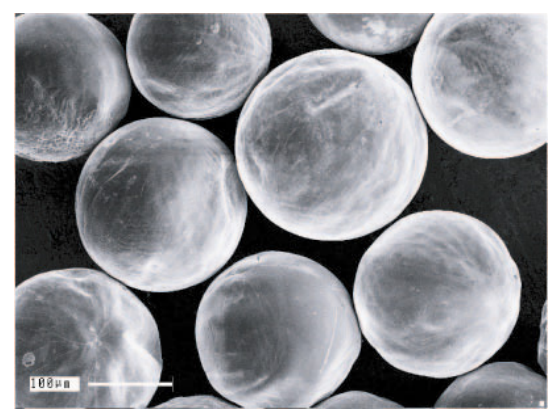

Figure1: Initial cooper powder.

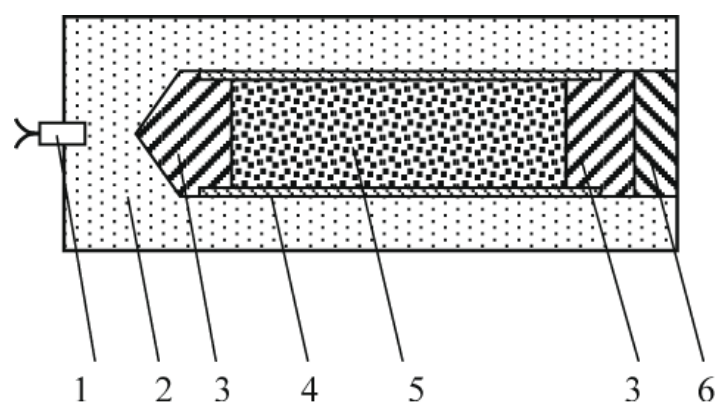

Figure 2: Scheme of explosive compaction: 1 - detonator, 2 explosive charge, 3 - steel plugs, 4 - container (steel tube $12 \mathrm{~mm}$ in diameter), 5 - copper powder, 6 - momentum trap.

Explosive compaction occurs under the action of the detonation products of the contact explosive charges. For varying of the detonation velocity the charges were made from ammonite, RDX and mixture of ammonite with RDX in different proportions. The detonation velocity (D) was measured by electrical contact technique, and ranged from 3.19 to 5.26 $\mathrm{km} / \mathrm{s}$. Container wall was thin compared to the thickness of explosive layer and diameter of the powder sample. Structure of compacts cross-sections were studied using an optical microscope NEOPHOT.

In Fig. 3 the structures of the cross sections near the axis of the samples is shown for three different values of detonation velocity $(3.19,3.95$ and $5.26 \mathrm{~km} / \mathrm{s})$ and at the same thickness of explosive charge $-5 \mathrm{~mm}$.

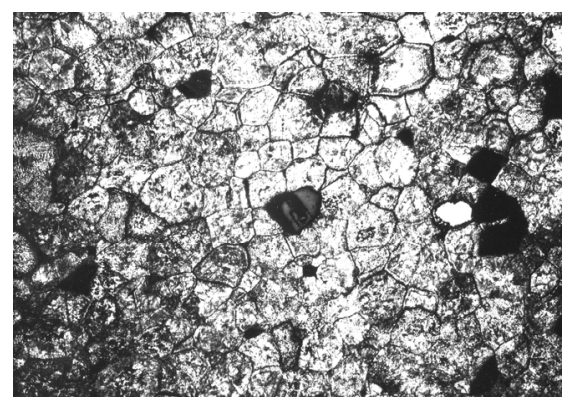

(a)

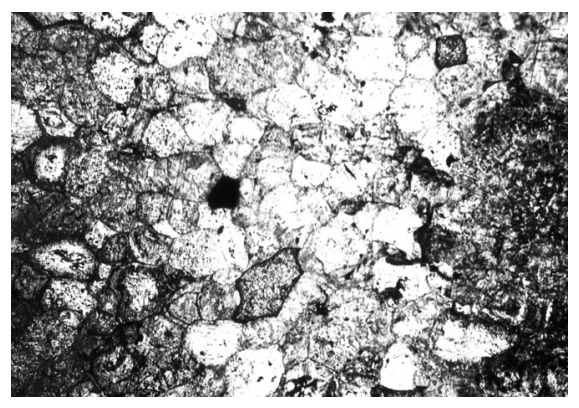

(b)

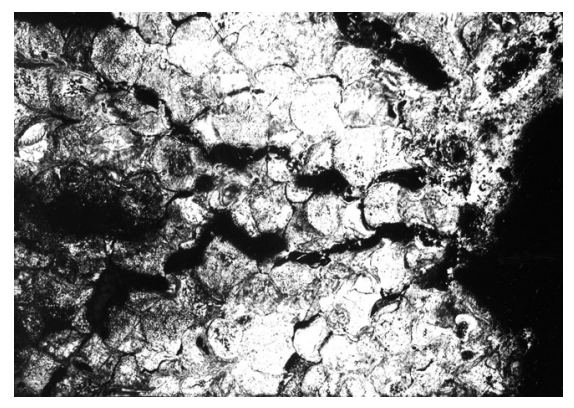

(c)

Figure 3: The structures of the cross sections of the samples for three different values of $D$ and at the same thickness of explosive charge $-5 \mathrm{~mm}$ : a) $D=3.19 \mathrm{~km} / \mathrm{s}$; b) $D=3.95 \mathrm{~km} / \mathrm{s}$; c) $D=5.26 \mathrm{~km} / \mathrm{s}$.

It can be noted that with the increase of the detonation velocity, and hence the shock pressure, a compacts structures change. At minimum value of $D$ compact is homogeneous. Then, in the center due to the irregular reflection of the converging shock wave a zone of melt appear. And at $D=5.26 \mathrm{~km} / \mathrm{s}$ in the compact the radial cracks arise. A further increase in $D$ can lead to the destruction of the container. To investigate the effect of the thickness of the explosive charge on the crack formation, an experiment was conducted in which the thickness of the charge was increased to $10 \mathrm{~mm}$, and the detonation velocity was $5.17 \mathrm{~km} / \mathrm{s}$. The structure of this compact is shown in Fig. 4. 


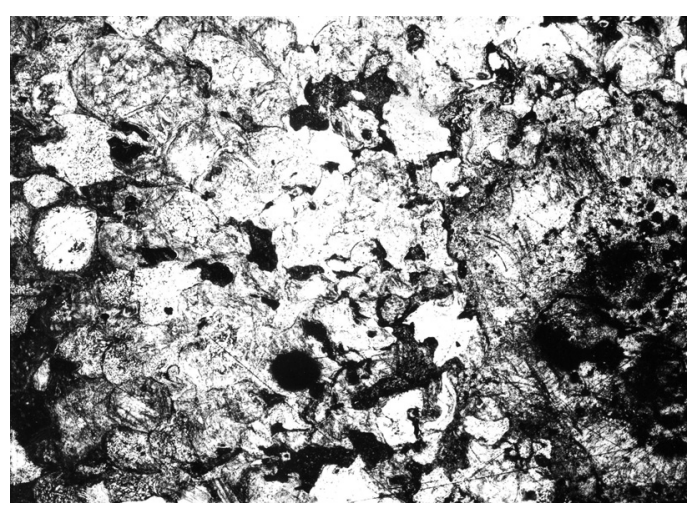

Figure 4: The structures of the cross section of the sample loaded at $D=5.12 \mathrm{~km} / \mathrm{s}$ and thickness of the explosive charge $10 \mathrm{~mm}$.

Comparing structures from Fig. $3 \mathrm{a}$ and Fig. 4, it is visible, that in spite of approximately identical detonation regimes, in a compact in Fig. 4 cracks are absent.

\section{NUMERICAL SIMULATION OF THE EXPLOSIVE LOADING}

$\mathrm{I}$

$\mathrm{n}$ order to gain a better insight into the effect of loading conditions and, in particular, to study the effect of detonation velocity, explosive thickness, and explosion pressure on the properties of the final sample, we numerically solved the problem about powder compaction in the axisymmetric case using conditions of above mentioned experiments.

The problem statement according to experimental scheme is clear from Fig. 5.

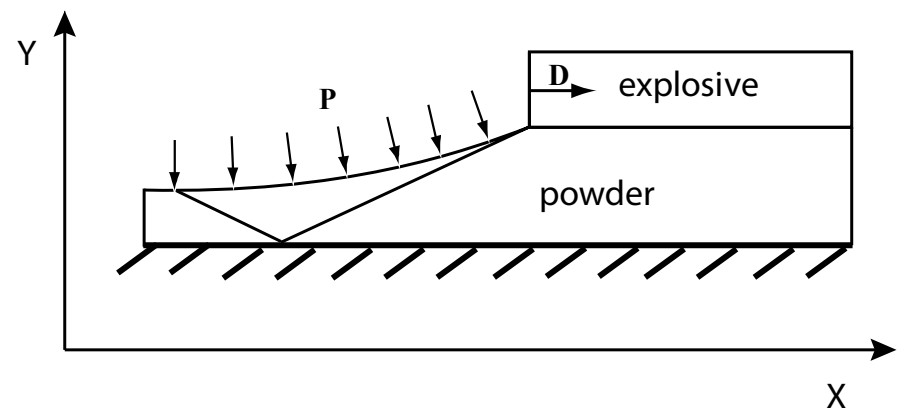

Figure 5: The problem statement.

We solved the full system of equations governing the deformation of a porous elastic-plastic material [3]. The action of the explosion products on the sample was modeled with a pressure applied to the upper border of the sample. The pressure was calculated by the approximation formula for the pressure upon unrestricted dispersion of detonation products [4]:

$$
P(t)=P_{H} \exp \left(-(t-x / D) / t_{1}\right), \quad t_{1}=\sqrt{\frac{3\left(\gamma_{e}+1\right)}{4\left(\gamma_{e}-1\right)}} \frac{\delta_{e}}{D}
$$

Here $\delta_{e}$ is the explosive thickness and $\gamma_{e}$ is the adiabatic exponent of the detonation products. Since the problem is symmetric, a half of the experimental assembly is considered.

The symmetry axis is the axis of the container with the powder. On the symmetry axis rigid wall boundary conditions are set. The right boundary is considered to be free of stress, and at the left boundary condition of a rigid wall is put. Computation of the contact boundaries is performed by using a symmetric algorithm [5]. The calculations are carried out by the M.L. Wilkins scheme [6]. The shock wave propagates from left to right. Geometric dimensions and values of the physical parameters correspond to the experimental data mentioned above. In this paper a few-parametric equation of state is applied [7], which has allowed to simulate shock-wave processes with a minimal number of physical parameters as the initial data. 


$$
\begin{aligned}
& E=E_{x}+c_{v, l} T+\frac{1}{2} c_{v, e 0} T^{2}\left(\frac{V}{V_{0}}\right)^{2 / 3} \\
& P=-\left(\frac{d E_{x}}{d V}\right)+\frac{\gamma_{l} c_{v, l} T}{V}+\frac{1}{3} \frac{c_{v, e 0} T^{2}}{V}\left(\frac{V}{V_{0}}\right)^{2 / 3}
\end{aligned}
$$

or, in terms of free energy

$$
F(V, T)=E_{x}(V)+c_{v, l} T \ln \left(\frac{\theta(V)}{T}\right)-\frac{1}{2} c_{v, e 0} T^{2}\left(\frac{V}{V_{0}}\right)^{2 / 3}
$$

where $P_{x}$ and $E_{x}$ - pressure and specific internal energy of the zero isotherm, $T$ - temperature, $c_{v}=c_{v, l}+c_{v, e}-$ heat capacity at constant volume, $\theta(V)$ - the Debye temperature.

The equation of state presented here is based on the dependence of the Gruneisen coefficient $\gamma$ on the volume [8]

$$
\begin{aligned}
& \gamma(V)=2 / 3-2 /\left(1-a V_{0} / V\right) \\
& a=1+2 /\left(\gamma_{s}-2 \beta\right)+2 P_{t, 0} / K_{s}
\end{aligned}
$$

where $\gamma_{s}=\beta K_{s} V_{0} / c_{v}, K_{s}$ - adiabatic bulk modulus, $\beta$-oefficient of thermal expansion, $P_{t, 0}$ - heat pressure under the normal conditions.

To find the elastic curves a generalized model describing the Gruneisen coefficient $\gamma(V)$ is used:

$$
\gamma(V)=-\left(\frac{2-t}{3}\right)-\frac{V}{2}\left[\frac{d^{2}\left(P_{x} V^{2 t / 3}\right) / d V^{2}}{d\left(P_{x} V^{2 t / 3}\right) / d V}\right]
$$

at $t=0$ the equation corresponds to the Landau and Slater theory [8,9], at $t=1$ it corresponds to the Dugdale and MacDonald hypothesis [10], and at $t=2$ to the theory of free volume [11].

In the physics of shock waves a method of calculating the pressure at the Hugoniot adiabat of the porous material by pressure on the "reference" Hugoniot adiabat of monolithic material [12] is known:

$$
P_{h, p}=\frac{P_{b}\left(1+0.5 \gamma(V)\left(1-V_{0} / V\right)\right)}{1+0.5 \gamma(V)\left(1-V_{00} / V\right)}
$$

Here $V$ is the specific volume of the Hugoniot adiabats, $V_{0}$ and $V_{00}$ are specific volumes of monolithic and porous materials, respectively, at the normal initial conditions.

\section{CALCULATION RESULTS AND DISCUSSION}

F Ig. 6, a and b shows the pressure isolines for the cases of planar and cylindrical symmetries with identical loading conditions. It is seen from Fig. 6 that, in the planar statement of the problem, a regular reflection of the incident shock wave takes place. In the case of the cylindrical loading scheme, the incident shock waves bends as it approaches the cylinder axis, and, under the same loading conditions, an irregular reflection occurs.

Fig. 7a shows the pressure profile near the symmetry axis for the cases of planar and cylindrical statements (solid and dashed curves, respectively). An appreciable pressure rise near the symmetry axis is observed in the case of cylindrical configuration compared to the planar problem due to the divergence of the shock wave to the axis.

Fig. $7 \mathrm{~b}$ shows the profile of the longitudinal velocity $u_{x}$ across the sample under loading behind the shock front. The solid and dashed lines show the data for the planar and axisymmetric problem statements, respectively. It is clearly seen that the velocity in the cylindrical case is much greater than in the planar variant.

As stated above, an important problem is preservation of finish compact, i.e., preventing its mechanical failure and obtaining a sample with uniform properties. Using criterion (3), we can find the interface between the solid and distructed materials. The regions of the compacted and porous materials for various explosive thicknesses for the external pressures 
$P=0.05 \mathrm{Mbar}$ and $P=0.075 \mathrm{Mbar}$ are shown in Fig. 8 , a and b, respectively. In these calculations, the detonation velocity was $D=5 \mathrm{~km} / \mathrm{s}$. The solid, dashed, and dot-and-dash lines outline the destruction regions for the explosive thicknesses $\delta_{e}=2 \mathrm{~cm}, \delta_{e}=3 \mathrm{~cm}$, and $\delta_{e}=5 \mathrm{~cm}$, respectively. Region 1 is the compacted region, and Region 2, the destruction region. An analysis of these graphs shows that an increase in the explosive thickness and, hence, an increase of the loading decay time does not cause any substantial shrinkage of the destruction region.
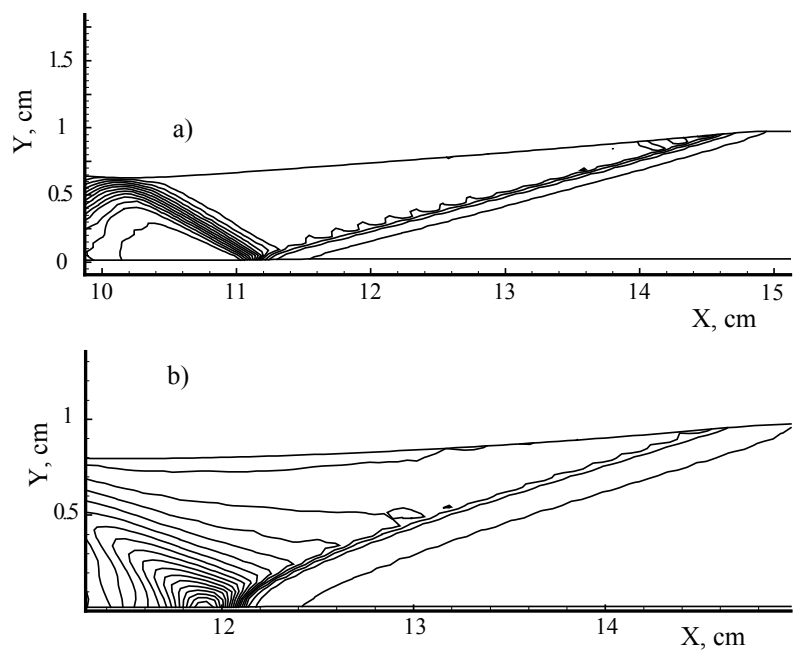

Figure 6: Pressure isolines: a) planar geometry; b) cylindrical configuration.
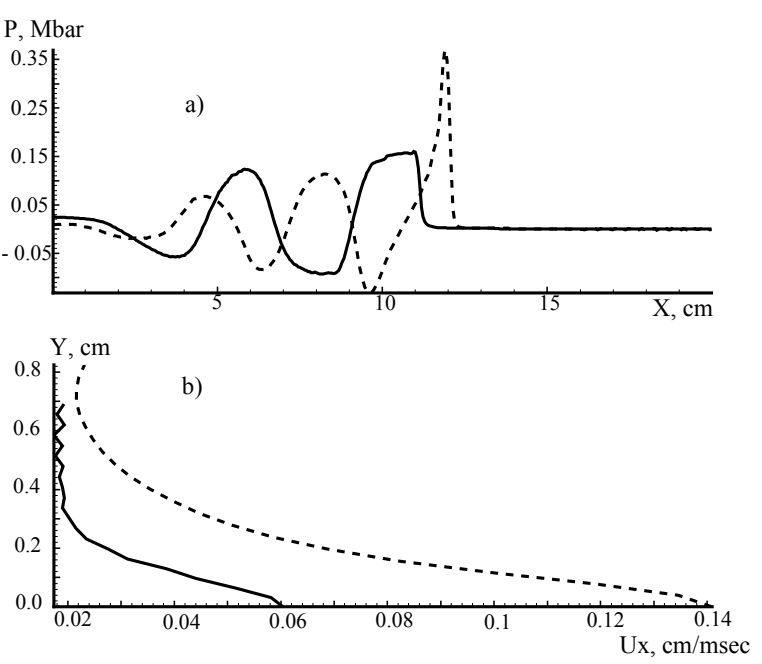

Figure 7: Pressure profile (a) and longitudinal-velocity profile $u_{x}(y)$ (b) for the planar and cylindrical geometries (solid and dashed lines, respectively).
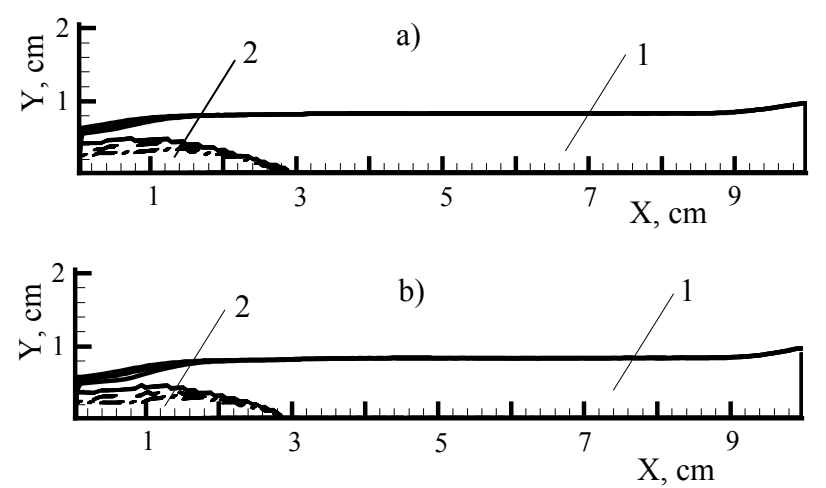

Figure 8: Compacted and destruction regions for various explosive thicknesses under external pressures $P=0.05$ Mbar (a) and $P=0.075$ Mbar (b). The detonation velocity is $D=5 \mathrm{~km} / \mathrm{s}$. The solid, dashed, and dot-and-dash lines refer to the explosive thicknesses $\delta_{e}=2 \mathrm{~cm}, \delta_{e}=3 \mathrm{~cm}$, and $\delta_{e}=5 \mathrm{~cm}$, respectively. The compacted and destruction regions are indicated by 1 and 2 .

It should be emphasized that this conclusion is valid for criterion (3). In derivation of (3), it was implicitly assumed that the interfacial melted zones are narrow, and the material in these zone rapidly solidifies as the particles in the bulk of the material undergo cooling. If this condition does not hold, then there can be a situation in which, by the moment of arrival of the unloading wave, the material in the interfacial zones still remains melted, which will prevent compaction. In this case, the dimensions of the destruction region will be dependent on the loading decay time and on the explosive thickness. The explosive thickness should be large enough to prevent shock wave damping in the powder and to enable complete pore collapsing in the sample. Fig. 9, a and b shows the density isolines for the explosive thickness $\delta_{e}=0.5 \mathrm{~cm}$ and the external pressure $P=0.05$ Mbar. Parts a and b of Fig. 9 depict the data for the axisymmetric and planar problem statements. Damping of the incident shock wave is evident from the figure. This results in incomplete powder compaction; the latter is clear from Fig. 10, which shows the distribution of porosity $m_{1}$ across the sample. The solid and dashed lines in this figure correspond to the planar case and to the cylindrical configuration, respectively. An analysis of 
these graphs shows that, in the axisymmetric case, due to the wave divergence to the axis, the pores undergo collapsing in a larger volume than in the planar variant.
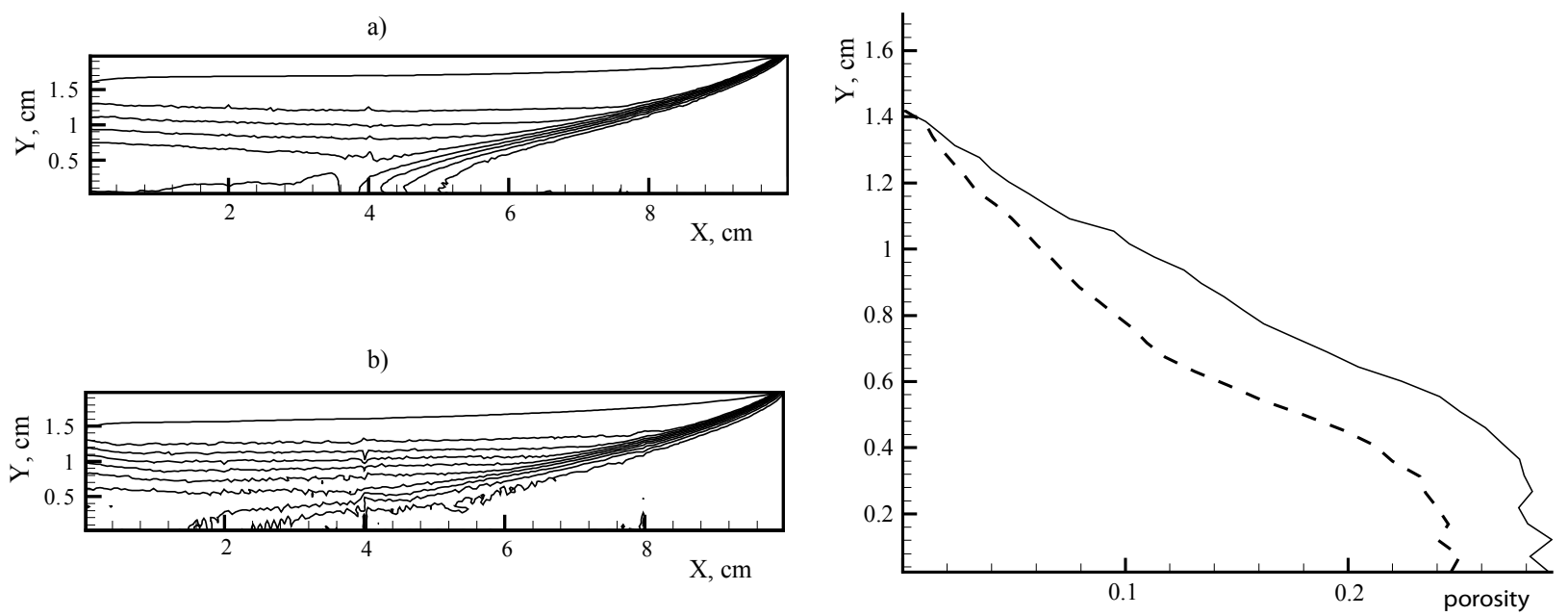

Figure 9: Density isolines for $\delta_{e}=0.5 \mathrm{~cm}$ : a) cylindrical configuration; b) planar statement.

Figure 10: Porosity $m_{1}$ for $\delta_{e}=0.5 \mathrm{~cm}$ : solid line is planar statement; dashed line is cylindrical configuration.

A twofold increase in the explosive thickness makes the decay of the incidence shock wave less intensive. The density isolines for the explosive thickness $\delta_{e}=1 \mathrm{~cm}$ and the external pressure $P=0.05 \mathrm{Mbar}$ are shown in Fig. 11, a and b. Parts $\mathrm{a}$ and $\mathrm{b}$ of this figure shows the calculation data for the axisymmetric and planar statements, respectively. It is clearly seen that in the case of cylindrical symmetry the shock wave bends near the axis, giving rise to an irregular reflection; in the planar configuration, a regular interaction occurs. Fig. 12, which depicts the distribution of porosity $m_{1}$ across the sample compacted in the cylindrical geometry (the dashed line in Fig. 12), is indicative of complete collapsing of pores over the entire thickness of the sample. In the planar case (see the dashed line in Fig. 12), the complete collapsing of pores is observed approximately over half the thickness of the sample, and the porosity near the symmetry axis is close to the initial one, $m_{1}^{0}$.
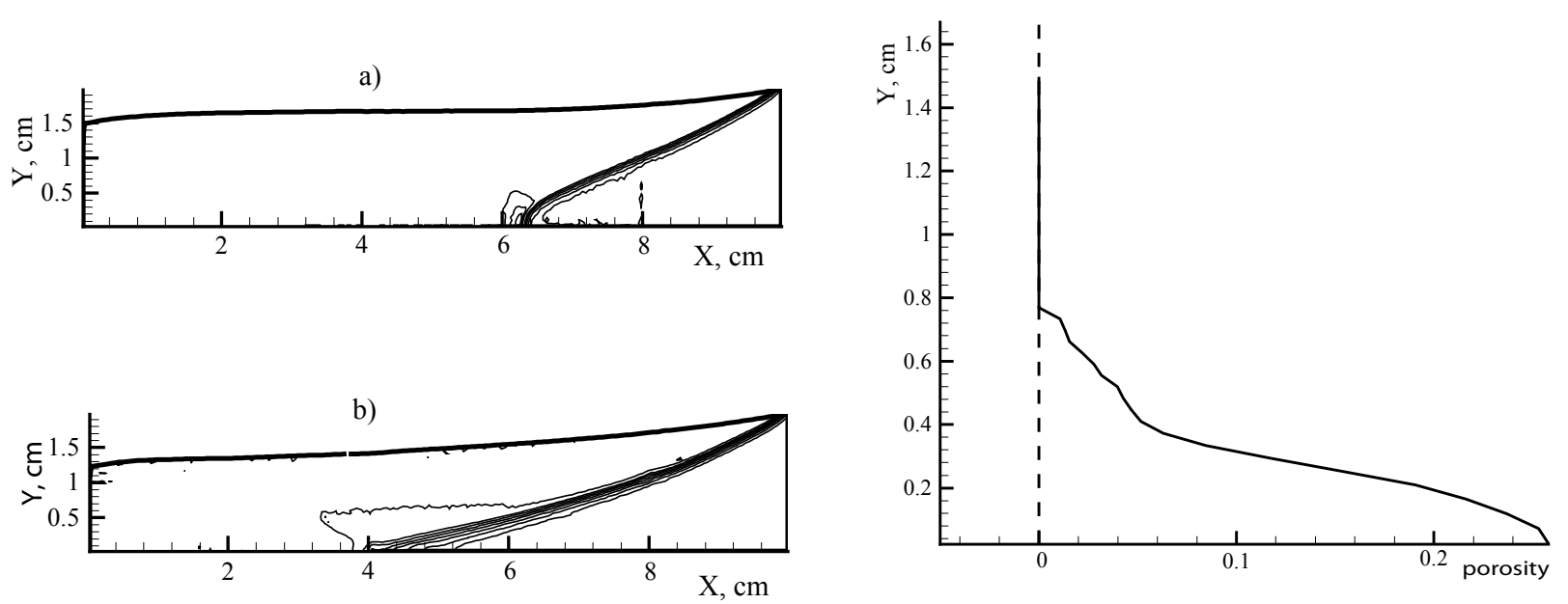

Figure 11: Density isolines for $\delta_{e}=1 \mathrm{~cm}$ : a) cylindrical configuration; b) planar configuration.

Figure 12: Porosity $m_{1}$ for $\delta_{e}=1 \mathrm{~cm}$ : solid line - planar statement; dashed line - cylindrical configuration.

The density isolines for the explosive thickness $\delta_{e}=2 \mathrm{~cm}$ are shown in Fig. 13, a and b. The external pressure was taken to be $P=0.05$ Mbar. Parts a and $\mathrm{b}$ of this figure show the calculation data for the axisymmetric and planar statements. In the cylindrical case (see Fig. 13, a), an irregular reflection is clearly observed, whereas in the planar case (see Fig. 13, b) the 
incident shock wave interacts with the rigid wall in the regular manner. In both cases, all pores in the sample collapse completely.

Further calculations were carried out for the explosive thicknesses $\delta_{e}=2 \mathrm{~cm}, \delta_{e}=3 \mathrm{~cm}$ and $\delta_{e}=5 \mathrm{~cm}$.

Fig. 14, a and b illustrates the effect of applied pressure on the thickness of the destruction region. In the calculations, the external pressures were $P=0.05 \mathrm{Mbar}$ and $P=0.075 \mathrm{Mbar}$, respectively, and the detonation velocity in both cases was $D=7 \mathrm{~km} / \mathrm{s}$. The solid and dashed lines show the data for the explosive thicknesses $\delta_{e}=3 \mathrm{~cm}$ and $\delta_{e}=5 \mathrm{~cm}$. Regions 1 and 2 are the compacted and destruction regions. As is seen from the figure, an increase in the external load causes no shrinkage of the destruction zone. Thus, it can be concluded that an increase in the decay time of the pressure applied to the sample resulting from an increase in the explosive thickness or in the value of the external load does not make the destruction zone shrink at a fixed propagation velocity of the detonation wave.

a)

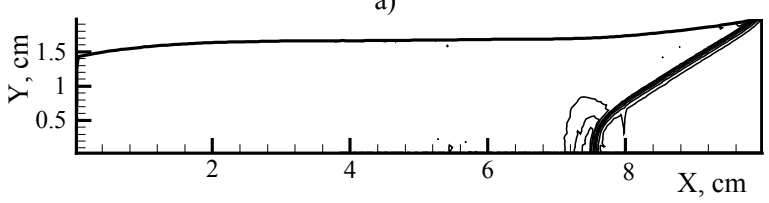

b)

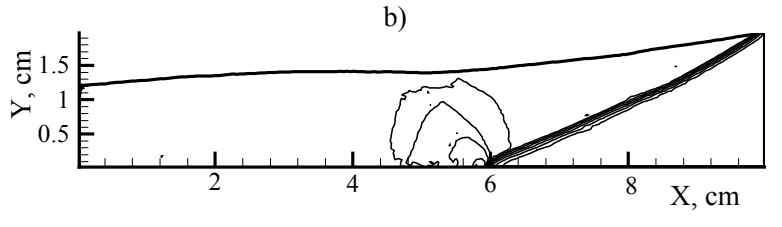

Figure 13: Density isolines for $\delta_{e}=2 \mathrm{~cm}$ : a) cylindrical configuration; b) planar statement.

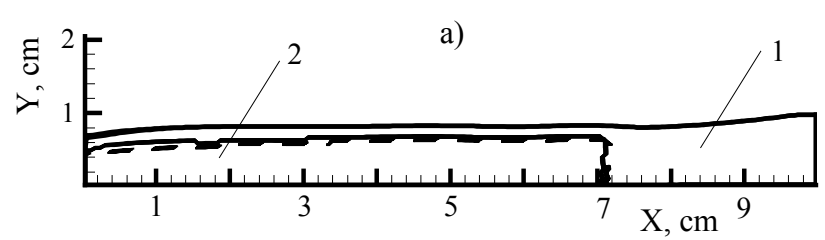

b)

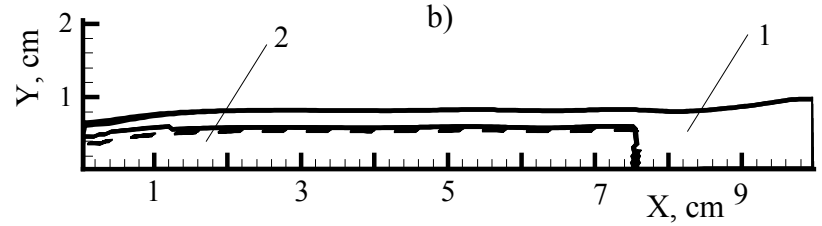

Figure 14: Compacted and destructed regions for two values of external pressure, $P=0.05 \mathrm{Mbar}(\mathrm{a})$ and $P=0.075 \mathrm{Mbar}$ (b). The detonation velocity is $D=7 \mathrm{~km} / \mathrm{s}$. The solid and dashed lines show the calculation data for the explosive thicknesses $\delta_{e}=3 \mathrm{~cm}$ and $\delta_{e}=5 \mathrm{~cm}$.

As shown by above mentioned experiments an increase of the velocity of the detonation wave results in a considerable shrinkage of the destruction region. Fig. 15 show the compacted (1) and destructed (2) regions in the sample for the detonation velocities $D=3,5,7 \mathrm{~km} / \mathrm{s}$ at a fixed explosive thickness $\delta_{e}=5 \mathrm{~cm}$ and at a fixed external pressure $P=0.05$ Mbar. The solid, dashed, and dot-and-dash lines show the calculation data for the detonation velocities $D=3 \mathrm{~km} / \mathrm{s}$, $D=5 \mathrm{~km} / \mathrm{s}$, and $D=7 \mathrm{~km} / \mathrm{s}$.

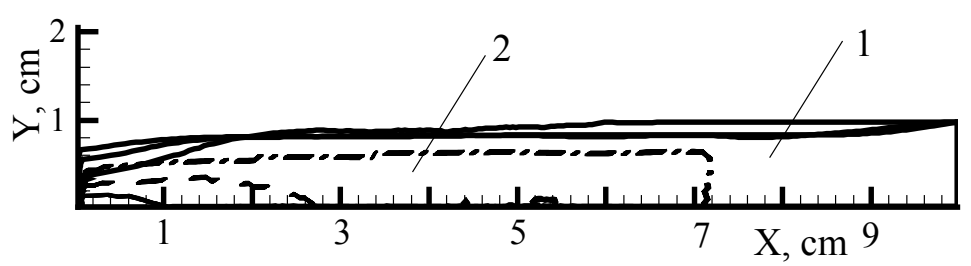

Figure 15: Compacted (1) and destructed (2) regions for three values of the detonation velocity. The solid, dashed, and dot-and-dash lines refer to $D=3 \mathrm{~km} / \mathrm{s}, D=5 \mathrm{~km} / \mathrm{s}$, and $D=7 \mathrm{~km} / \mathrm{s}$.

The isolines of pressure for the indicated loading parameters are shown in Fig. 16, a-c. It is seen from the graphs that, as the shock-wave propagation velocity increases, the angle of incidence decreases and the reflected shock causes material destruction (see Fig. 16, b and c). As the velocity of the detonation wave increases, the angle of incidence of the incident shock wave increases and, as it is seen from Fig. 16, a, at the velocity $D=3 \mathrm{~km} / \mathrm{s}$ the incident shock wave is close to the normal shock and the amplitude of the reflection wave is almost zero.

Since in the case of cylindrical symmetry no regular reflection occurs, the final sample turns out to be inhomogeneous. Fig. 17 shows the distribution of the longitudinal velocity $u_{x}$ (Fig. 17, a) and temperature $T$ (fig. 16, b) across the sample 
in the compacted region for the detonation velocity $D=5 \mathrm{~km} / \mathrm{s}$. An appreciable non-uniformity in the distribution of parameters is evident from the graphs. Near the axis, both the velocity and temperature are greater than in the region some distance away from it.
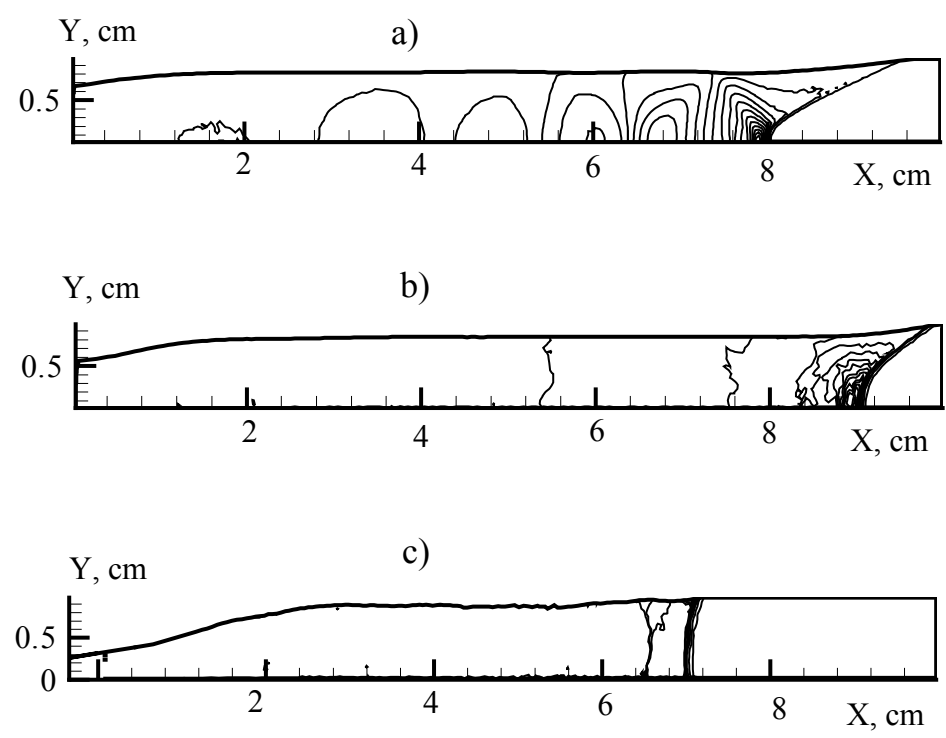

Figure 16: Pressure isolines: a) detonation velocity $D=7 \mathrm{~km} / \mathrm{s}$; b) detonation velocity $D=5 \mathrm{~km} / \mathrm{s}$; c) detonation velocity $D=3 \mathrm{~km} / \mathrm{s}$.
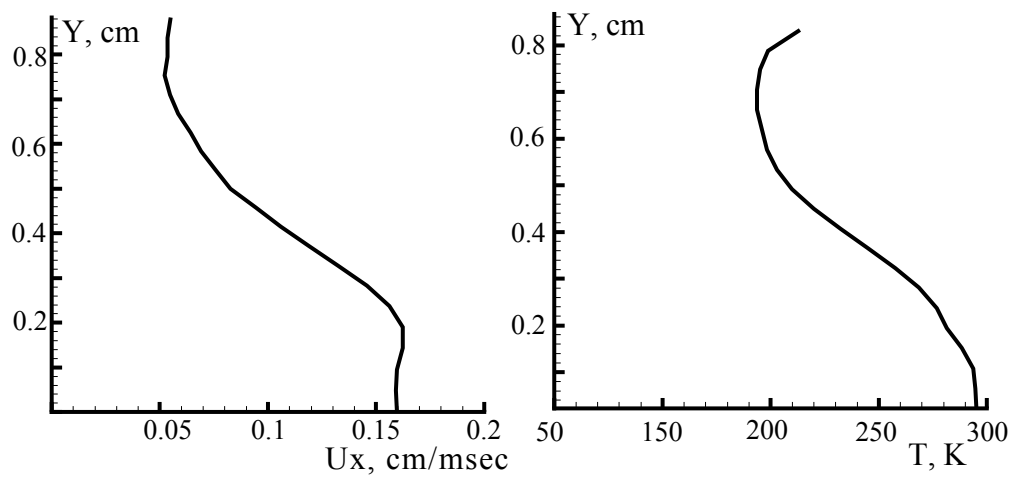

Figure 17: Predicted distributions of the longitudinal velocity $u_{x}$ (a) and temperature $T$ (b) across the compacted region of the sample for the detonation velocity $D=5 \mathrm{~km} / \mathrm{s}$.

Parts a and b of Fig. 18 show the distributions of the longitudinal velocity $u_{x}$ and temperature $T$ across the compacted region of the sample predicted for the detonation velocity $D=3 \mathrm{~km} / \mathrm{s}$. Here, under identical loading parameters, the final sample is quire homogeneous.

As a result, it becomes possible to obtain spatially uniform compacted samples. The necessary condition for this is sufficiently low detonation velocity, equal, for the aluminum powder, to $0.3 \mathrm{~cm} / \mathrm{msec}$. Here, on the one hand, compaction condition (1) should be fulfilled and, on the other, the uniformity of loading parameters across the sample should be ensured.

Thus, the compaction of powders with low detonation velocities results in a considerable shrinkage of destruction zones in finish samples and in spatial uniformity of material parameters in their compacted parts.

The performed analysis shows that an increase in the decay time of the pressure applied to the sample due to an increase of the explosive thickness or the external loading causes no shrinkage of the destructed region at a fixed propagation velocity of the detonation wave. Simultaneously, a decrease in the propagation velocity of the detonation wave results in an appreciable shrinkage of this region. 

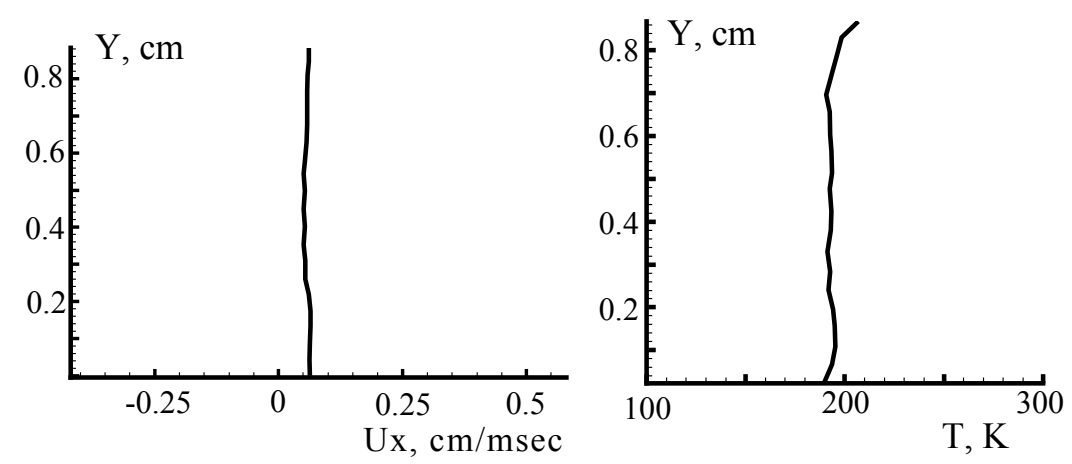

Figure 18: Distributions of the longitudinal velocity $u_{x}$ (a) and temperature $T$ (b) across the compacted region of the sample for the detonation velocity $D=3 \mathrm{~km} / \mathrm{s}$.

\section{CONCLUSIONS}

$\mathrm{J}$ oint theoretical and experimental studies have allowed to implement an approach that uses mathematical and physical simulation of shock-wave loading of powdered materials. A numerical simulation of shock wave propagation and deformation of the experimental assembly has been performed.

The temperature distributions over the sample thickness in the compacted zone for several values of detonation velocity show that at higher speeds there is considerable heterogeneity in the temperature distribution over the sample thickness. Near the axis of the sample the temperature has a higher value than in distance from it.

An increase in the pressure decay time due to increasing either the explosive thickness or the external loading intensity causes no shrinkage of the destruction zone at a fixed propagation velocity of the detonation wave. Compaction of powders with low detonation velocities results in a considerable shrinkage of destruction zones in finish samples and in a uniform distribution of material parameters in the compacted region.

\section{REFERENCES}

[1] V.F. Nesterenko, High-rate deformation of heterogeneous materials, Nauka, Novosibirsk (1992) (in Russian).

[2] R. Prümmer, Powder compaction. Explosive welding, forming and compaction, London; New York: Appl. Sci. Publ., (1983).

[3] S.P. Kiselev, V.M. Fomin, J. of Applied Mechanics and Technical Physics. 34(6) (1993) 861.

[4] V.V. Pai, G.E. Kuz'min, I.V. Yakovlev, Combustion, Explosion, and Shock Waves, 31(3) (1995) 124.

[5] A.I. Gulidov, I.I. Shabalin, Numerical Realization of the Boundary Conditions in Dynamic Contact Problems, Preprint ITAM SB RAS, Novosibirsk (1987) (in Russian).

[6] M. L. Wilkins, Computer simulation of dynamic phenomena, Springer, Berlin, Heidelberg (1999).

[7] E. I. Kraus, Vestnik NGU. Fizika, 2(2) (2007) 65 (in Russian).

[8] C. Slater, Introduction in the chemical physics.- New-York-London: McGraw Book company, Inc., (1935) 239.

[9] L. D. Landau, K.P. Stanyukovich, Dokl. Akad. Nauk SSSR, 46 (1945) 399 (in Russian).

[10] J. S. Dugdale, D. McDonald, Phys. Rev., 89 (1953) 832.

[11] V.Ya. Vaschenko, V.N. Zubarev, Sov. Phys. Solid State, 5 (1963) 653.

[12] R. G. McQueen, S. P. Marsh, J. W. Taylor, J. N. Fritz, High-Velocity Impact Phenomena, ed. by R. Kinslow, New York: Academic Press (1970) 293. 